\title{
Some Studies on the Lorentz Theory of Radiation Reaction in Relation to a Charged Particle Acted on by a Constant Force in a Finite Time Interval
}

\author{
Rajat Roy \\ Department of Electronics and Electrical Communication Engineering, Indian Institute of Technology, \\ Kharagpur, India \\ Email: rajatroy@ece.iitkgp.ernet.in
}

Received 17 January 2016; accepted 1 February 2016; published 4 February 2016

Copyright (c) 2016 by author and OALib.

This work is licensed under the Creative Commons Attribution International License (CC BY).

http://creativecommons.org/licenses/by/4.0/

(c) (i) Open Access

\begin{abstract}
An attempt to predict some experimental verification of the Lorentz theory of radiation reaction using theoretical methods is being made. The dynamics of charged particles using the equations of motion of this theory is compared to that of uncharged particles. Some verifiable consequence of pre-acceleration is also worked out.
\end{abstract}

Keywords

Lorentz Theory of Radiation Reaction, Experimental Verification

Subject Areas: Theoretical Physics

\section{Introduction}

It is well known fact that the theory of radiation reaction in electrodynamics as worked out by Lorentz [1] (actually by Lorentz, Abraham and Dirac) has solutions, which exhibits a runaway nature and/or non-causal preacceleration. In fact the non-causal pre-acceleration without runaway makes more sense and in this paper we will only be concerned with this solution of the equation of motion. It is generally believed that the non-causal pre-acceleration exists for a short duration (typically $10^{-23}$ seconds for the electron) when it becomes comparable in magnitude to that of the actual acceleration and hence can never be detected in an experiment. Below we study the case of a charged particle when it is moving under the influence of a constant force preferably of a non-electromagnetic origin for a finite time duration and compare it with the dynamics of an uncharged particle 
of equal mass under the action of the same force. The second particle of course does not experience radiation reaction. Also we put a different initial condition and try to find out if there is some measurable parameter which detects pre-acceleration in an experiment. In the last section we discuss some difficulties with the attempts to eliminate pre-acceleration theoretically as they exist in literature [2]. Only non-relativistic dynamical equations have been used for the present and that too for one dimensional motion.

\section{The Dynamical Equation of the Charged Particle with Some Initial Condition and Acted on by a Constant Force $f_{\text {ext }}$ from 0 to $t$ Seconds}

From Equation (1.1) of ref. [1] we can write the equation of motion in one dimension as

$$
m a=f_{\text {ext }}+m \tau_{0} \frac{\mathrm{d} a}{\mathrm{~d} T},
$$

where $\tau_{0}$ is about $10^{-23}$ sec. for the electron and $m$ is its mass. From Equations (1.2) and (1.3) of the same reference we can write the pre accelerated solution without runaway as

$$
a(T)=\frac{\mathrm{e}^{T / \tau_{0}}}{m \tau_{0}} \int_{T}^{\infty} \mathrm{e}^{-T^{\prime} / \tau_{0}} f_{e x t}\left(T^{\prime}\right) \mathrm{d} T^{\prime} .
$$

For the special case of $f_{\text {ext }}$ to be a constant $f_{0}$ between $T=0$ and $T=t$ we can represent it mathematically in terms of the unit step function $u(T)$ as

$$
f_{e x t}=f_{0}[u(T)-u(T-t)],
$$

where $u(T)=0$ for $T<0$ and $u(T)=1$ for $T>0$. We also prefer $f_{\text {ext }}$ to be of non electromagnetic origin so that it can act equally well on charged and uncharged particles alike. This restriction can be relaxed when uncharged particles are not under consideration as we see in Section 3. Thus for $f_{\text {ext }}$ of the type described by Equation (3) the acceleration $a(T)$ from Equation (2) becomes

$$
a(T)=\frac{f_{0}}{m}\left[\mathrm{e}^{T / \tau_{0}}(1-u(T))+u(T)\right]-\frac{f_{0}}{m}\left[\mathrm{e}^{(T-t) / \tau_{0}}(1-u(T-t))+u(T-t)\right]
$$

The time derivative of acceleration and the velocity respectively are

$$
\frac{\mathrm{d} a}{\mathrm{~d} T}=\frac{f_{0}}{m}\left[\frac{\mathrm{e}^{T / \tau_{0}}}{\tau_{0}}(1-u(T))+\delta(T)\left(1-\mathrm{e}^{T / \tau_{0}}\right)\right]-\frac{f_{0}}{m}\left[\frac{\mathrm{e}^{(T-t) / \tau_{0}}}{\tau_{0}}(1-u(T-t))+\delta(T-t)\left(1-\mathrm{e}^{(T-t) / \tau_{0}}\right)\right]
$$

and

$$
\begin{aligned}
v(T)= & \frac{f_{0}}{m}\left[\tau_{0} \mathrm{e}^{T / \tau_{0}}(1-u(T))+\int_{-\infty}^{T} \delta\left(T^{\prime}\right) \tau_{0} \mathrm{e}^{T / \tau_{0}} \mathrm{~d} T^{\prime}+T u(T)\right] \\
& -\frac{f_{0}}{m}\left[\tau_{0} \mathrm{e}^{(T-t) / \tau_{0}}(1-u(T-t))+\int_{-\infty}^{T} \delta\left(T^{\prime}-t\right) \tau_{0} \mathrm{e}^{\left(T^{\prime}-t\right) / \tau_{0}} \mathrm{~d} T^{\prime}+(T-t) u(T-t)\right]+K \text { (constant) } .
\end{aligned}
$$

The constant $K$ will be zero if $v(-\infty)=0$. For the case of the uncharged particle of the same mass which we have already assumed to be accelerated by the same force $f_{e x t}$ in accordance with Newton's laws the velocity is

$$
v_{u n}(T)=\frac{f_{0}}{m}[T u(T)-(T-t) u(T-t)]+K^{\prime} \quad \text { (constant), }
$$

where the subscript un of $v$ denotes that it is the velocity of the uncharged particle. For $v_{u n}(-\infty)=0$ we again get the constant $K^{\prime}=0$. With this set of initial conditions $v(-\infty)=v_{u n}(-\infty)=0$ it is easy to show that the final velocity attained at $T \geq t$ is given identically for the two particles to be

$$
v(t)=v_{u n}(t)=\frac{f_{0} t}{m} .
$$

Thus in the Lorentz theory of radiation reaction the change in kinetic energy of a radiating charged particle is 
equal to that of a non radiating uncharged particle when acted on by equal external forces in equal intervals of time. This fact can be determined in an experiment. The energy radiated out by the charged particle is given by the Larmor's formula

$$
m \tau_{0} \int_{0}^{t} a^{2}(T) \mathrm{d} T \approx \frac{f_{0}^{2}}{m} \tau_{0} t
$$

One can easily show that this comes from the extra work done by $f_{\text {ext }}$ when it is applied on the charged particle as compared to when it is applied on the uncharged one in the time interval 0 to $t$.

\section{Possible Means to Experimentally Detect Pre-Acceleration}

In this section we study only the dynamics of charged particles so the restriction on $f_{\text {ext }}$ to be of non electromagnetic origin may be relaxed. The initial condition is changed from $v(-\infty)=0$ to some finite velocity. Let us for example take the initial velocity to be the same as that of the final velocity attained by the particle in the last section that is $v(-\infty)=f_{0} t / m$. This becomes the new value of the constant $K$ in Equation (6). Now we use the energy conservation equation given by Gron (see Equation (2.1) of ref. [1]) in the non relativistic limit

$$
\boldsymbol{v} \cdot \boldsymbol{f}_{\text {ext }}=\frac{\mathrm{d} E_{k}}{\mathrm{~d} T}-\boldsymbol{v} \cdot \boldsymbol{f}_{A}
$$

In the non relativistic limit $\boldsymbol{f}_{A}$ is the Schott force and for the one dimensional problem of Section 2 and the initial condition $v(-\infty)=f_{0} t / m=K$ for the time $T<0$ we have

$$
\frac{\mathrm{d} E_{k}}{\mathrm{~d} t}=v f_{\mathrm{A}}=v m \tau_{0} \frac{\mathrm{d} a}{\mathrm{~d} T} .
$$

From these and using the values of $\frac{\mathrm{d} a}{\mathrm{~d} T}$ and $v$ from Equations (5) and (6) we get the value of the change in kinetic energy from $-\infty$ to zero to be

$$
\Delta E_{k}=m \tau_{0} \int_{-\infty}^{0} v \frac{\mathrm{d} a}{\mathrm{~d} T} \mathrm{~d} T \approx \frac{f_{0}^{2}}{m} \tau_{0} t .
$$

Since this change in kinetic energy of the particle beam before $T<0$ is almost equal to the magnitude of the radiated energy in the interval $0 \leq T \leq t$ that is during the interval when a constant force is applied (compare Equations (9) and (12)), it must be experimentally detectable. This can be done by spectral methods to detect the shift in kinetic energy of the electrons (particles) in the beam when measured at $T=0$ as compared to the value much before this time. In fact it will confirm or disprove the Lorentz, Abraham and Dirac theory of radiation reaction and the pre acceleration associated with it.

\section{Theoretical Attempts to Cancel Pre-Acceleration in the Lorentz Theory of Radiation Reaction}

Yaghjian [2] had tried to modify the existing equation of motion of the Lorentz theory of radiation reaction in a way such that pre-acceleration is excluded. He writes (see Equation (8.34) of Ref. [2]) in place of Equation (1) of the present paper the following equation of motion expressed as per the notations used in this paper

$$
m a=f_{\text {ext }}+m \tau_{0} u(T) \frac{\mathrm{d} a}{\mathrm{~d} T}
$$

This will first of all violate energy conservation as expressed by Equation (10) and there is no simple way to get around this difficulty if other aspects of Lorentz theory are to be kept intact after $T=0$. Also the solution given for this equation of motion which is stated as

$$
\begin{gathered}
a(T)=0 \text { for } T<0 \\
a(T)=-\frac{1}{m} \int_{T}^{\infty} f_{\text {ext }}\left(T^{\prime}\right) \frac{\mathrm{d}}{\mathrm{d} T^{\prime}}\left[\mathrm{e}^{-\frac{1}{\tau_{0}} \int_{T}^{T^{\prime}} \frac{\mathrm{d} T^{\prime \prime}}{u\left(T^{\prime \prime}\right)}}\right] \mathrm{d} T^{\prime} \text { for } T \geq 0
\end{gathered}
$$


has some features which makes it behave differently from the expression for acceleration as given by Equation (2) near $T=0$. This is because the function $u\left(T^{\prime \prime}\right)$ in the expression above rapidly falls towards zero as $T^{\prime \prime} \rightarrow 0$ causing some sort of "overshoot" if the force is of the form given by Equation (3) and may have some experimentally observed consequences especially when $t$ (please refer to the previous sections) is small.

\section{References}

[1] Gron, O. (2012) Electrodynamics of Radiating Charges. Advances in Mathematical Physics, 2012, Article ID: 528631. http://dx.doi.org/10.1155/2012/528631

[2] Yaghjian, A.D. (1992) Relativistic Dynamics of a Charged Sphere. Springer-Verlag, New York. 